\title{
Isolated omental panniculitis in a child with abdominal pain. Case report
}

\author{
Asst. Prof. Mustafa O. Oztan, M.D. ${ }^{a}$, Assoc. Prof. Tunc Ozdemir, M.D. ${ }^{b}$, Melek Uncel, M.D. ${ }^{c}$, \\ Assoc. Prof. Gulden Diniz, M.D ${ }^{c}$ and Prof. Dr. Gokhan Koyluoglu, M.D. ${ }^{a}$
}

\begin{abstract}
Isolated omental panniculitis is a rare entity mostly seen in adults. It presents with the inflammation of the fatty tissue of the omentum. The symptoms may vary from local (e.g. abdominal tenderness or palpable mass) to systemic manifestations including abdominal pain, back pain, fever, weight loss and bowel disturbances. We presented this case as a first awareness of omental panniculitis in a child which must be kept in mind at the differential diagnosis of ileus so that unnecessary surgeries might be avoided.

Key words: panniculitis, omentum, child.
\end{abstract}

http:/ / dx.doi.org/10.5546/aap.2016.eng.e425

\section{INTRODUCTION}

Panniculitis is a rare disease of unknown etiology that is characterized by chronic inflammation of the adipose tissue in the abdomen. The most affected site in the abdomen is the small bowel mesentery, but it can also infrequently involve other fatty tissues (retroperitoneal, mesocolonic, omental, peripancreaticor pelvic). ${ }^{1}$ We herein present a case with an isolated omental panniculitis in a child diagnosed by exploratory laparotomy. Our objective is to create awareness about this little known disease and if possible, to avoid unnecessary surgery.

a. Department of Pediatric Surgery, Katip Celebi University Faculty of Medicine. Izmir, Turkey.

b. Department of Pediatric Surgery, Tepecik Research and Education Hospital. Izmir, Turkey.

c. Laboratory of Pathology, Tepecik Research and Education Hospital. Izmir, Turkey.

E-mail Address:

Mustafa O. Oztan, Asst. Prof., M.D.: mustafaonur.oztan@ikc.edu.tr

Funding: None.

Conflict of interest: None.

Received: 2-19-2016

Accepted: 6-27-2016

\section{CASE REPORT}

A 13-year-old boy presented with a history of epigastric pain and vomiting lasting for two days. His medical history revealed appendectomy 9 months ago. The omentum was detached from the tip of the appendix and appendectomy was done at this time without omentectomy.

The physical examination revealed a lowgrade fever $\left(37.5^{\circ} \mathrm{C}\right)$ and a severe tender palpable mass in the epigastrium. There was leukocytosis $(20,500 / \mu \mathrm{L})$, high levels of erythrocyte sedimentation rate (ESR- $99 \mathrm{~mm} / \mathrm{h}$ ) and C-reactive protein (CRP-145 mg/dL) at the laboratory tests. Plain abdominal X-ray was normal. Abdominal ultrasonography revealed a hyperechoic mass in the epigastrium around the colon. Contrast enhanced computed abdominal tomography (CAT) showed soft tissue mass $(51 \times 30 \mathrm{~mm})$ at the anterior surface of the transverse colon according to the omentum, which had marked heterogeneity and inflammatory changes (Figure 1).

FIGURE 1: CAT scan

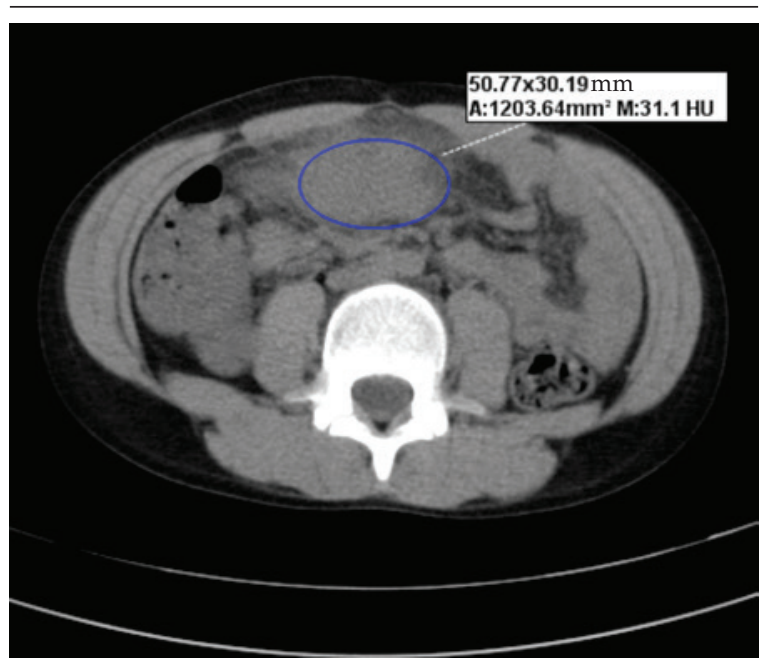

Axial contrast enhanced CAT scan demonstrates a large $(51 \mathrm{~mm} \times 30 \mathrm{~mm})$, moderately heterogeneous, well marginated mass (circle) above the transverse colon. The lesion contains mixed soft-tissue- and fat-density material with a higher density than subcutaneous fat. 
The patient was hospitalized and followed conservatively with intravenous fluids, nasogastric suction and nil per oral. Organized abscess, intestinal duplication, lipomatous tumors, extraintestinal stromal tumors and omental torsion were considered as possible diagnoses. Due to the continued existence of acute abdomen findings and vomiting, an exploratory open laparotomy was performed on the next day instead of laparoscopy because of the tumor possibility noted on the CAT scan. During the surgical procedure, a firm conglomerate of the omentum on the transverse colon, without compression or narrowing of the intestinal lumen has been observed (Figure 2). The mass was excised from the colon with no residue or perforation. The patient was discharged from hospital after 3 days uneventfully.

Histopathological examination of the specimen revealed a chronic inflammatory process including plasma cells, lymphocytes and foamy macrophages. Fibrous septa surrounding the fat lobules and mixed inflammatory infiltrate and huge myofibroblasts in the septa with extension into the periphery of the fat lobules were also seen. Immunohistochemical staining showed abundant macrophages that tended to cluster in the septa with anti-CD68 antibody (Figure 3a-d). All these findings were consistent with omental panniculitis.

FIGURE 2: Intraoperative view of the mass

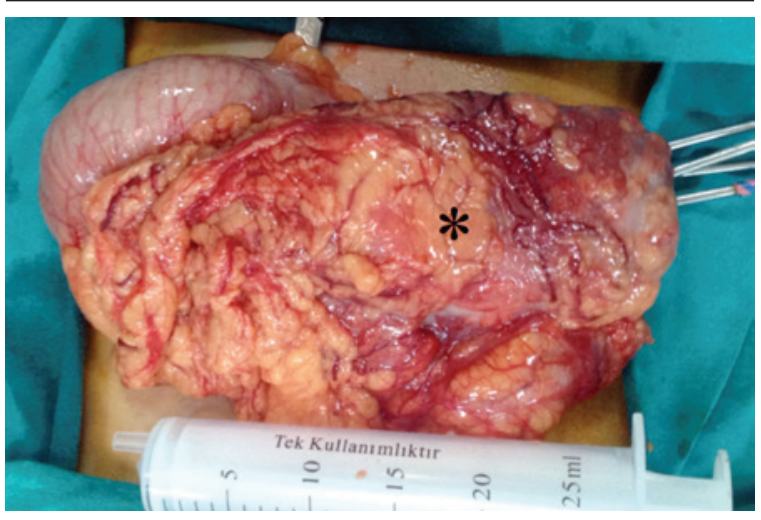

Intraoperative aspect of omental panniculitis above the transverse colon. A hypervascularized, fatty, rubbery mass presented as a nodular heterogeneous tissue (asterisk), which was strongly attached to the colon. There were no ischemic changes nor adhesions. The mass was resected without complications.

\section{DISCUSSION}

The term "panniculitis" defines inflammation of the fat in the subcutaneous tissues and can be caused by a number of factors; including lymphedema, immune-mediated, infectious, trauma-induced or malignancies. The intraabdominal involvement of this pathology at the mesentery was first named by Jura in 1924 as "retractile mesenteritis" and later in the 1960's labeled by Ogden as "mesenteric panniculitis". ${ }^{2}$

Katz et al. have described these lesions as intraabdominal panniculitis, which is characterized as: 1 . Diffuse, single, or multiple mass like fatty lesions in the mesentery, retroperitoneum, omentum, and/or pelvis; 2 . Histological confirmation of fat necrosis with inflammatory infiltrate and/or infiltration with foamy lipid-laden macrophages; and 3. No evidence of pancreatitis, inflammatory bowel disease, extraabdominal fat necrosis (Weber-Christian disease). ${ }^{3}$

The etiology of intraabdominal panniculitis is not clear. Specific etiologies in various cases have been reported as a possible cause including concurrent or remote infection, recent prior surgery, healing appendicitis, drugs, prior abdominal trauma, vascular problems like mesenteric thrombosis or mesenteric arteriopathy, ulcerative disease, and retained suture material. ${ }^{1,3,4}$ Most cases are idiopathic and there is no coexistent disease or prior intervention.

\section{FIGURE 3: Histopathologic examination}

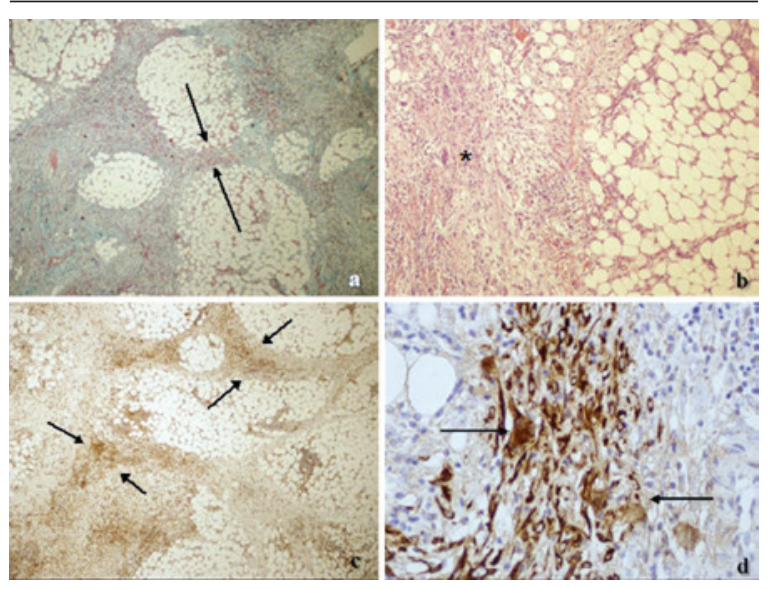

Fibrous septa surrounding the fat lobules (between the arrows) (Gomori's Trichrome, x40) (a). Mixed inflammatory infiltrate and huge myofibroblasts in the septa with extension into the periphery of the fat lobules (asterisk) (H\&E, x200) (b). Macrophages were abundant and tend to cluster in the septa with anti-CD68 antibody (between the arrows) (DAB, x40) (c). The huge myofibroblasts positive for anti-pancytokeratin antibody (arrows) (DAB, x400) (d). 
In up to $90 \%$ of cases, intraabdominal panniculitis involves the small bowel mesentery or sigmoid mesentery (mesenteric panniculitis), but on rare occasions, the region of involvement is the mesocolon, omentum, retroperitoneum or pelvis. $^{5}$

Mesenteric panniculitis is a progressive disease and has three typical grades according to histological grading: mesenteric lipodystrophy (when fatty degeneration and necrosis are predominant), mesenteric panniculitis (subsequent marked inflammatory reaction), retractile mesenteritis or mesenteric fibrosis (fibrosis of the adipose tissue). ${ }^{5}$

Mesenteric panniculitis is usually asymptomatic and often incidental. Daskalogiannaki et al. reported an incidence of $0.6 \%$ of mesenteric panniculitis among 7620 patients on CAT scan evaluation. ${ }^{6}$ But the patients may present with either local abdominal tenderness or a palpable abdominal mass or systemic manifestations like fever, weightloss, abdominal pain, back pain and bowel symptoms (vomiting, constipation, diarrhea, rectal bleeding) of variable duration. ${ }^{7}$ The most cases reported in the literature are adults. Viswanathan and Murray reported a single case of a 6 year old girl and reviewed another 16 published pediatric cases of mesenteric panniculitis. ${ }^{8}$

Isolated omental panniculitis is rare subgroup of intraabdominal panniculitis, in which the omentum is the only site affected without evidence of pancreatitis, inflammatory bowel disease, or extraabdominal fat necrosis. ${ }^{4,9}$ There are only 4 cases of intraabdominal panniculitis with isolated omental involvement reported in medical literature, all in adult patients. In one of these cases the medical treatment contributed to the cause of the panniculitis, while the other three were idiopathic. ${ }^{10-13}$

There is no consensus on the management of patients with intraabdominal panniculitis. Drug therapy is not standardized and a variety of anti-inflammatory, immunomodulatory and antifibrotic agents are used according to the stage of the disease. ${ }^{12}$ If medical therapy fails or in the last phase when extensive fibrosis causes life threatening complications such as bowel obstruction or perforation; surgical intervention is mandatory. ${ }^{13}$

At the former appendectomy operation of our patient, the affected omentum was slightly attached to the appendix, and was not removed. We usually perform a partial omentectomy at the appendectomy operation when the omentum is markedly swollen and/or infected. We do not recommend an extensive omentectomy because of the protective role of the omentum from intestinal adhesions, which is also reported in the article of Ariake et al. ${ }^{14}$ It is possible that the omentum we do not resected at the first operation may be the cause of the panniculitis, but there is no enough evidence for resection of the affected omentum in order to prevent a subsequent panniculitis. The etiology of intraabdominal panniculitis is still subject to speculation, which is mentioned at the previous published articles.

To define a lesion as "panniculitis", the histologic diagnosis must be confirmed definitely. These lesions are defined on CAT scan as lesions with a fibrous capsule containing areas of fat density separated with areas of water or soft-tissue density. They may be closely adjacent to the gut indistinguishable from a mass arising from the intestine. ${ }^{4}$ Because the omental involvement is extremely rare, the prior 3 adult patients with isolated omental panniculitis were operated because of abdominal tenderness or suspected malignant diseases. ${ }^{11-13}$ In the latter reported case, Jeon et al. have decided to take a CT-guided biopsy based on the CAT scan features of the mass (presence of highattenuating mesenteric fat and tumoral pseudo capsule) and treated the patient with low dose prednisolone. ${ }^{10}$ We operated the patient because of the persistence of abdominal tenderness, vomiting and possible malignant origin. But if we and the radiologist have thought a possible diagnosis of omental panniculitis, so we could make a laparoscopic approach and take frozen section biopsies or plan a CAT/US guided biopsy as suggested in the articles of Jeon et al. and Alkubeyyer et al. ${ }^{10,15}$ After the confirmation of the diagnosis, follow up with antibiotics and anti-inflammatory drugs would be more appropriate for this patient.

In conclusion, the exact diagnosis of omental panniculitis is very difficult because of the rarity of the disease in children. Although one of the most frequent reasons for recurrent abdominal pain and vomiting after surgery is adhesive intestinal obstruction, we think that these patients should be also imaged with ultrasonography to help the diagnosis and if necessary with CAT scan either. We presented this case as a first, in order to increase awareness of omental panniculitis in children, so that unnecessary surgeries might be avoided. 


\section{REFERENCES}

1. Emory TS, Monihan JM, Carr NJ, Sobin LH. Sclerosing mesenteritis, mesenteric panniculitis and mesenteric lipodystrophy: a single entity? Am J Surg Pathol 1997;21(4):392-8.

2. Jura SV. Mesenterile retrattile e sclerosante. Policlinica (Sez Prat) $1924 ; 31: 575$.

3. Issa I, Baydoun H. Mesenteric panniculitis: various presentations and treatment regimens. World J Gastroenterol 2009;15(30):3827-30.

4. Katz ME, Heiken JP, Glazer HS, Lee JK. Intraabdominal panniculitis: clinical, radiographic, and CT features. AJR Am J Roentgenol 1985;145(2):293-6.

5. Rumman N, Rumman G, Sharabati B, Zagha R, et al. Mesenteric panniculitis in a child misdiagnosed as appendicular mass: a case report and review of literature. Springerplus 2014;3:73.

6. Daskalogiannaki M, Voloudaki A, Prassopoulos P, MagkanasE, etal.CT evaluation of mesenteric panniculitis: prevalence and associated diseases. AJR Am J Roentgenol 2000;174(2):427-31

7. Gunduz Y, Tatli AP, Kara RO. Mesenteric panniculitis: a case report and review of the literature. Maedica (Buchar) 2012;7(4):344-7.

8. Viswanathan V, Murray KJ. Idiopathic sclerosing mesenteritis in paediatrics: report of a successfully treated case and a review of literature. Pediatr Rheumatol Online J 2010;8:5.
9. Hirono S, Sakaguchi S, Iwakura S, Masaki K, et al. Idiopathic isolated omental panniculitis. JClin Gastroenterol 2005;39(1):79-80.

10. Jeon EJ, Cho SM. Idiopathic isolated omental panniculitis confirmed by percutaneous CT-guided biopsy. Gut Liver 2009;3(4):321-4.

11. Rozin A, Bishara B, Ben-Izhak O, Fischer D, et al. Fibrosing omental panniculitis and polyserositis associated with long-term treatment by paroxetine. Isr Med Assoc J 2000; 2(9):714-6.

12. Lheureux $\mathrm{P}$, Matos $\mathrm{C}$, Charlier $\mathrm{PH}$, Van Romphey A, et al. Omental panniculitis: an unusual cause of acute appendiceal syndrome. Ann Emerg Med 1987;16(2):224-6.

13. Gu GL, Wang SL, Wei XM, Ren L, et al. Sclerosing mesenteritis as a rare cause of abdominal pain and intraabdominal mass: a cases report and review of the literature. Cases J 2008;1(1):242.

14. Ariake K, Yokoyama S, Doi T, Takemura S, et al. Effect of omentum removal on the risk for postoperative adhesive small bowel obstruction recurrence: a case-control study. Int J Surg 2015;13:27-32.

15. Alkubeyyer MA, Alfuhaid TR. Mesenteric panniculitis. Imaging of a rare cause of chronic abdominal pain. Saudi Med J 2007;28(2):276-8. 